\title{
A Proof of Simultaneous Linearization with a Polylog Estimate
}

by

\author{
Tomoki KAWAHIRA
}

Presented by Stanisław KWAPIEN

Summary. We give an alternative proof of simultaneous linearization recently shown by T. Ueda, which connects the Schröder equation and the Abel equation analytically. In fact, we generalize Ueda's original result so that we may apply it to the parabolic fixed points with multiple petals. As an application, we show a continuity result on linearizing coordinates in complex dynamics.

1. Introduction. Let us start with a worked out example to explain the motivation to consider the simultaneous linearization theorem.

Cauliflowers. In the family of quadratic maps, the simplest parabolic fixed point is given by $g(w)=w+w^{2}$ (whose Julia set is called the cauliflower). Now we consider its perturbation of the form $f(w)=\lambda w+w^{2}$ with $\lambda \nearrow 1$. According to [Mi, $\S 8$ and $\S 10]$, we have the following fact:

Proposition 1.1 (Königs and Fatou coordinates). Let $K_{f}$ and $K_{g}$ be the filled Julia sets of $f$ and $g$. Then we have the following:

(1) There exists a unique holomorphic branched covering map $\phi_{f}: K_{f}^{\circ} \rightarrow \mathbb{C}$ satisfying the Schröder equation $\phi_{f}(f(w))=\lambda \phi_{f}(w)$ and $\phi_{f}(0)=$ $\phi_{f}(-\lambda / 2)-1=0$. The map $\phi_{f}$ is univalent near $w=0$.

(2) There exists a unique holomorphic branched covering map $\phi_{g}: K_{g}^{\circ} \rightarrow \mathbb{C}$ satisfying the Abel equation $\phi_{g}(g(w))=\phi_{g}(w)+1$ and $\phi_{g}(-1 / 2)=0$. The map $\phi_{g}$ is univalent on a disk $|w+r|<r$ with small $r>0$.

Note that $-\lambda / 2$ and $-1 / 2$ are the critical points of $f$ and $g$ respectively.

2000 Mathematics Subject Classification: 37F99, 30D05. point.

Key words and phrases: simultaneous linearization, polylogarithm, parabolic fixed 
ObSeRvation. Set $\widetilde{w}=\phi_{f}(w)$. Now the proposition above asserts that the action of $\left.f\right|_{K_{f}^{\circ}}$ is semiconjugate to $\widetilde{w} \mapsto \lambda \widetilde{w}$ by $\phi_{f}$. Consider the Möbius map

$$
W=S_{f}(\widetilde{w})=\lambda(\widetilde{w}-1) /(\lambda-1) \widetilde{w}
$$

that sends $\{0,1, \lambda\}$ to $\{\infty, 0,1\}$ respectively. By taking conjugation by $S_{f}$, the action of $\widetilde{w} \mapsto \lambda \widetilde{w}$ is viewed as $W \mapsto W / \lambda+1$. Set $W=\Phi_{f}(w):=$ $S_{f} \circ \phi_{f}(w)$. Now we have

$$
\Phi_{f}(f(w))=\Phi_{f}(w) / \lambda+1 \quad \text { and } \Phi_{f}(-\lambda / 2)=0 .
$$

On the other hand, by setting $W=\Phi_{g}(w):=\phi_{g}(w)$, we can view the action of $\left.g\right|_{K_{g}^{\circ}}$ as $W \mapsto W+1$. Thus we have

$$
\Phi_{g}(g(w))=\Phi_{g}(w)+1 \quad \text { and } \Phi_{g}(-1 / 2)=0 .
$$

If $\lambda$ tends to 1 , that is, $f \rightarrow g$, the semiconjugate action in $W$-coordinate converges uniformly on compact sets. Now it would be natural for $\Phi_{f}$ to tend to $\Phi_{g}$. However, as one can see by referring to the proof of the proposition in [Mi, $\S 8$ and $\S 10], \phi_{f}$ and $\phi_{g}$ are given in completely different ways, and thus we cannot conclude the convergence $\Phi_{f} \rightarrow \Phi_{g}$ a priori.

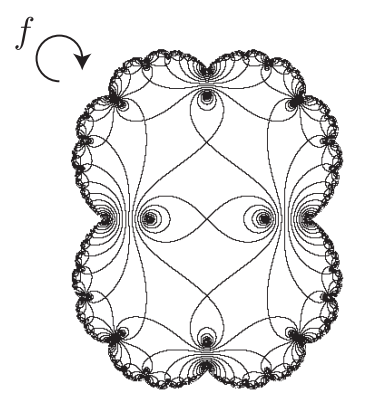

$$
\Phi_{f} \downarrow
$$

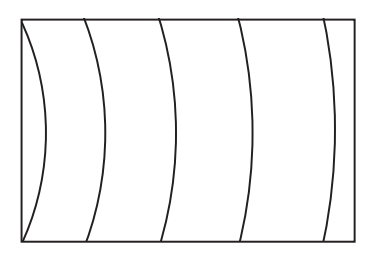

$W \mapsto W / \lambda+1$

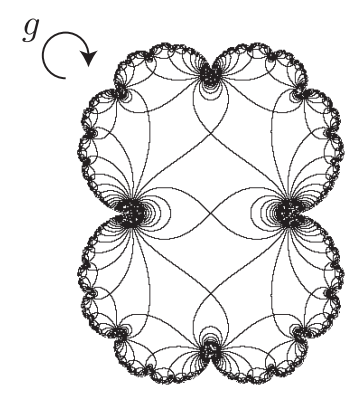

$\Phi_{g} \downarrow$

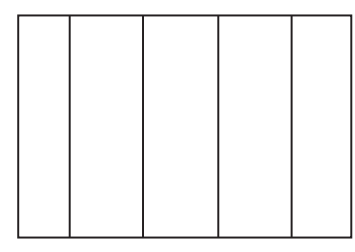

$W \mapsto W+1$

Fig. 1. Semiconjugation inside the filled Julia sets of cauliflowers

But there is another evidence that supports this observation. Figure 1 shows the equipotential curves of $\phi_{f}$ and $\phi_{g}$ in the filled Julia sets. We can find similar patterns and it seems that one converges to the other. Actually, we have the following: 
THEOREM 1.2. For any compact set $E \subset K_{g}^{\circ}$,

(1) $E \subset K_{f}^{\circ}$ for all $f \approx g$;

(2) $\Phi_{f} \rightarrow \Phi_{g}$ uniformly on $E$ as $f \rightarrow g$.

Here $f \approx g$ means that $f$ is sufficiently close to $g$, equivalently, $\lambda$ is sufficiently close to 1 . (See [Ka, Theorem 5.5] for a more general version of this proposition.) The proof of this theorem is given in Section 5, by using the simultaneous linearization theorem.

2. Simultaneous linearization. In this section we state the simultaneous linearization theorem. We first generalize the cauliflower setting above:

Perturbation of parabolics. Let $f$ be an analytic map defined on a neighborhood of 0 in $\widehat{\mathbb{C}}$ which is tangent to identity at 0 . That is, $f$ near 0 is of the form

$$
f(w)=w+A w^{m+1}+O\left(w^{m+2}\right)
$$

where $A \neq 0$ and $m \in \mathbb{N}$. By making a linear coordinate change $w \mapsto A^{1 / m} w$, we may assume that $A=1$. In the theory of complex dynamics such germs appear when we consider iteration of local dynamics near parabolic periodic points, and play very important roles. (See $[\mathrm{Mi}, \S 10]$ and $[\mathrm{Sh}]$ for example.) Now we consider a perturbation $f_{\varepsilon} \rightarrow f$ of the form

$$
f_{\varepsilon}(w)=\Lambda_{\varepsilon} w\left(1+w^{m}+O\left(w^{m+1}\right)\right)
$$

with $\Lambda_{\varepsilon} \rightarrow 1$ as $\varepsilon \rightarrow 0$. By taking branched coordinate changes $z=$ $-\Lambda_{\varepsilon}^{m} /\left(m w^{m}\right)$ and setting $\tau_{\varepsilon}:=\Lambda_{\varepsilon}^{-m}$, we have

$$
\begin{aligned}
f_{\varepsilon}(z) & =\tau_{\varepsilon} z+1+O\left(|z|^{-1 / m}\right) \\
\rightarrow f_{0}(z) & =z+1+O\left(|z|^{-1 / m}\right)
\end{aligned}
$$

uniformly near $w=\infty$ on the Riemann sphere $\widehat{\mathbb{C}}$. The simultaneous linearization theorem will give partially linearizing coordinates of $f_{\varepsilon}$ that depend continuously on $\varepsilon$ when $\tau_{\varepsilon} \rightarrow 1$ non-tangentially to the unit circle.

Let us formalize non-tangential access to 1 in the complex plane: After C. McMullen, for a variable $\tau \in \mathbb{C}$ converging to 1 , we say $\tau \rightarrow 1$ radially (or more precisely, $\alpha$-radially) if $|\arg (\tau-1)| \leq \alpha$ for some fixed $\alpha \in[0, \pi / 2$ ).

Ueda's modulus. Consider a continuous family $\left\{\tau_{\varepsilon}\right\}$ of complex numbers with $\varepsilon \in[0,1]$ such that $\left|\tau_{\varepsilon}\right| \geq 1$ and $\tau_{\varepsilon} \rightarrow 1 \alpha$-radially as $\varepsilon \rightarrow 0$. For simplicity we assume that $\tau_{\varepsilon}=1$ iff $\varepsilon=0$. Set $\ell_{\varepsilon}(z):=\tau_{\varepsilon} z+1$, which is an isomorphism of the Riemann sphere $\widehat{\mathbb{C}}$. If $\varepsilon>0$, then $b_{\varepsilon}:=1 /\left(1-\tau_{\varepsilon}\right)$ is a repelling fixed point of $\ell_{\varepsilon}$ with $\ell_{\varepsilon}(z)-b_{\varepsilon}=\tau_{\varepsilon}\left(z-b_{\varepsilon}\right)$. Thus the function

$$
N_{\varepsilon}(z):=\left|z-b_{\varepsilon}\right|-\left|b_{\varepsilon}\right|
$$


has the uniform increase property

$$
N\left(\ell_{\varepsilon}(z)\right)=\left|\tau_{\varepsilon}\right| N(z)+\frac{\left|\tau_{\varepsilon}\right|-1}{\left|\tau_{\varepsilon}-1\right|} \geq\left|\tau_{\varepsilon}\right| N(z)+\cos \alpha .
$$

Similarly, if $\varepsilon=0$, the function

$$
N_{0}(z):=\sup \left\{\operatorname{Re}\left(e^{i \theta} z\right):|\theta|<\alpha\right\}
$$

also has the corresponding property

$$
N_{0}\left(\ell_{0}(z)\right) \geq N_{0}(z)+\cos \alpha .
$$

In both cases, set

$$
\mathbb{V}_{\varepsilon}(R):=\left\{z \in \mathbb{C}: N_{\varepsilon}(z) \geq R\right\}
$$

for $R>0$. One can check that $N_{\varepsilon}(z) \leq|z|$ and

$$
\mathbb{V}_{\varepsilon}(R) \subset \mathbb{B}(R):=\{z \in \mathbb{C}:|z| \geq R\} \quad \text { for all } \varepsilon \in[0,1] .
$$

We will establish:

Theorem 2.1 (Simultaneous linearization). Let $\left\{f_{\varepsilon}: \varepsilon \in[0,1]\right\}$ be a family of holomorphic maps on $\mathbb{B}(R)$ such that as $\varepsilon \rightarrow 0$ we have the uniform convergence on compact sets of the form

$$
\begin{aligned}
f_{\varepsilon}(z) & =\tau_{\varepsilon} z+1+O\left(1 /|z|^{\sigma}\right) \\
\rightarrow f_{0}(z) & =z+1+O\left(1 /|z|^{\sigma}\right)
\end{aligned}
$$

for some $\sigma \in(0,1]$ and $\tau_{\varepsilon} \rightarrow 1 \alpha$-radially. If $R \gg 0$, then:

(1) For any $\varepsilon \in[0,1]$ there exists a holomorphic map $u_{\varepsilon}: \mathbb{V}_{\varepsilon}(R) \rightarrow \mathbb{C}$ such that

$$
u_{\varepsilon}\left(f_{\varepsilon}(z)\right)=\tau_{\varepsilon} u_{\varepsilon}(z)+1 \text {. }
$$

(2) For any compact set $K$ contained in $\mathbb{V}_{\varepsilon}(R)$ for all $\varepsilon \in[0,1], u_{\varepsilon} \rightarrow u_{0}$ uniformly on $K$.

This theorem is a mild generalization of Ueda's theorem in [Ue1] that deals with the case of $\sigma=1$. (See also [Ue2].) This plays a crucial role to show the continuity of tessellation of the filled Julia set for hyperbolic and parabolic quadratic maps. See [Ka]. C. McMullen showed that there exist quasiconformal linearizations with much wider domain of definition. In fact, $\tau_{\varepsilon} \rightarrow 1$ may be tangent to the unit circle (horocyclic in his terminology). See [Mc, §8].

Remark on the domain of convergence. We can take such a compact subset $K$ as above in

$$
\begin{aligned}
\Pi(R): & =\mathbb{C}-\left\{e^{\theta i} z: \operatorname{Re} z<R,|\theta| \leq \alpha\right\} \\
& =\left\{z \in \mathbb{C}: \operatorname{Re}\left(z-R^{\prime}\right) \geq\left|z-R^{\prime}\right| \sin \alpha\right\},
\end{aligned}
$$

which is a closed sector at $z=R^{\prime}=R / \cos \alpha>0$. In fact, for any $R>0$ and $\varepsilon \in[0,1], \Pi(R)$ is contained in $\mathbb{V}_{\varepsilon}(R)$. One can check this as follows. The 
complement of $\mathbb{V}_{\varepsilon}(R)$ is contained in $\left\{e^{\theta i} z: \operatorname{Re} z<R, \theta=\arg \left(-b_{\varepsilon}\right)\right\}$. Since $\left|\arg \left(-b_{\varepsilon}\right)\right| \leq \alpha$, we have the claim.

In the next section we give a proof of Theorem 2.1 that is also an alternative proof of Ueda's simultaneous linearization when $\sigma=1$. His original proof given in [Ue1] uses a technical difference equation which makes the proof beautiful and the statement a little more detailed. Here we present a simplified proof based on the argument of [Mi, Lemma 10.10] (its idea can be traced back at least to Leau's work on the Abel equation [L]) and an estimate on polylogarithm functions given in Section 4 .

3. Proof of the theorem. Let us start with a couple of lemmas. Set $\delta:=(\cos \alpha) / 2>0$. We first check:

LEMMA 3.1. If $R \gg 0$, there exists $M>0$ such that $\left|f_{\varepsilon}(z)-\left(\tau_{\varepsilon} z+1\right)\right| \leq$ $M /|z|^{\sigma}$ on $\mathbb{B}(R)$ and $N_{\varepsilon}\left(f_{\varepsilon}(z)\right) \geq N_{\varepsilon}(z)+\delta$ on $\mathbb{V}_{\varepsilon}(R)$ for any $\varepsilon \in[0,1]$.

Proof. The first inequality and the existence of $M$ are obvious. By replacing $R$ by a larger one, we have $\left|f_{\varepsilon}(z)-\left(\tau_{\varepsilon} z+1\right)\right| \leq M / R^{\sigma}<\delta$ on $\mathbb{B}(R)$. Then

$$
N_{\varepsilon}\left(f_{\varepsilon}(z)\right) \geq N_{\varepsilon}\left(\ell_{\varepsilon}(z)\right)-\delta \geq N_{\varepsilon}(z)+\delta .
$$

Let us fix such an $R \gg 0$. Then the lemma above implies that $f_{\varepsilon}\left(\mathbb{V}_{\varepsilon}(R)\right)$ $\subset \mathbb{V}_{\varepsilon}(R)$. Moreover, since $N_{\varepsilon}(z) \leq|z|$, we have

$$
\left|f_{\varepsilon}^{n}(z)\right| \geq N_{\varepsilon}\left(f_{\varepsilon}^{n}(z)\right) \geq N_{\varepsilon}(z)+n \delta \geq R+n \delta \rightarrow \infty .
$$

Thus $\mathbb{V}_{\varepsilon}(R)$ is contained in the basin of infinity and uniformly attracted to $\infty$ in the spherical metric of $\widehat{\mathbb{C}}$. In particular, this convergence to $\infty$ is uniform on $\Pi(R)$ for any $\varepsilon$.

Next we show a key lemma for the theorem:

LEMMA 3.2. There exists $C>0$ such that for any $\varepsilon \in[0,1]$ and $z_{1}, z_{2} \in$ $\mathbb{B}(2 S)$ with $S>R$, we have

$$
\left|\frac{f_{\varepsilon}\left(z_{2}\right)-f_{\varepsilon}\left(z_{1}\right)}{z_{2}-z_{1}}-\tau_{\varepsilon}\right| \leq \frac{C}{S^{1+\sigma}} .
$$

Proof. Set $g_{\varepsilon}(z):=f_{\varepsilon}(z)-\left(\tau_{\varepsilon} z+1\right)$. For any $z \in \mathbb{B}(2 S)$ and $w \in$ $\mathbb{D}(z, S):=\{w:|w-z|<S\}$, we have $|w|>S$. This implies $\left|g_{\varepsilon}(w)\right| \leq$ $M /|w|^{\sigma}<M / S^{\sigma}$ and thus $g_{\varepsilon}$ maps $\mathbb{D}(z, S)$ into $\mathbb{D}\left(0, M / S^{\sigma}\right)$. By the Cauchy integral formula (or the Schwarz lemma), it follows that $\left|g_{\varepsilon}^{\prime}(z)\right| \leq\left(M / S^{\sigma}\right) / S$ $=M / S^{1+\sigma}$ on $\mathbb{B}(2 S)$.

Let $\left[z_{1}, z_{2}\right]$ denote the oriented line segment from $z_{1}$ to $z_{2}$. If $\left[z_{1}, z_{2}\right]$ is contained in $\mathbb{B}(2 S)$, the inequality easily follows from

$$
\left|g_{\varepsilon}\left(z_{2}\right)-g_{\varepsilon}\left(z_{1}\right)\right|=\left|\int_{\left[z_{1}, z_{2}\right]} g_{\varepsilon}^{\prime}(z) d z\right| \leq \int_{\left[z_{1}, z_{2}\right]}\left|g_{\varepsilon}^{\prime}(z)\right||d z| \leq \frac{M}{S^{1+\sigma}}\left|z_{2}-z_{1}\right|
$$


with $C:=M$. Otherwise we have to take a roundabout way to get the estimate. Consider a circle with diameter $\left[z_{1}, z_{2}\right]$. Then $\left[z_{1}, z_{2}\right]$ divides the circle into two semicircles, and at least one of them is contained in $\mathbb{B}(2 S)$; denote it by $\left\{z_{1}, z_{2}\right\}$. Then

$$
\left|g_{\varepsilon}\left(z_{2}\right)-g_{\varepsilon}\left(z_{1}\right)\right|=\left|\int_{\left\{z_{1}, z_{2}\right\}} g_{\varepsilon}^{\prime}(z) d z\right| \leq \int_{\left\{z_{1}, z_{2}\right\}}\left|g_{\varepsilon}^{\prime}(z)\right||d z| \leq \frac{M}{S^{1+\sigma}} \cdot \frac{\pi}{2}\left|z_{2}-z_{1}\right|
$$

and the lemma holds with $C:=M \pi / 2(>M)$ for any $z_{1}, z_{2} \in \mathbb{B}(2 S)$.

Proof of Theorem 2.1. Set $z_{n}:=f_{\varepsilon}^{n}(z)$ for $z \in \mathbb{V}_{\varepsilon}(2 R)$. Note that $\left|z_{n}\right| \geq$ $N_{\varepsilon}\left(z_{n}\right) \geq 2 R+n \delta$ by (3.1). Now we fix $a \in \mathbb{V}_{\varepsilon}(2 R)$ and define $\phi_{n, \varepsilon}=\phi_{n}$ : $\mathbb{V}_{\varepsilon}(2 R) \rightarrow \mathbb{C}(n \geq 0)$ by

$$
\phi_{n}(z):=\frac{z_{n}-a_{n}}{\tau_{\varepsilon}^{n}} .
$$

For example, one can take such an $a$ in $\Pi(2 R)$ independently of $\varepsilon$. Then

$$
\left|\frac{\phi_{n+1}(z)}{\phi_{n}(z)}-1\right|=\left|\frac{z_{n+1}-a_{n+1}}{\tau_{\varepsilon}\left(z_{n}-a_{n}\right)}-1\right|=\frac{1}{\left|\tau_{\varepsilon}\right|} \cdot\left|\frac{f_{\varepsilon}\left(z_{n}\right)-f_{\varepsilon}\left(a_{n}\right)}{z_{n}-a_{n}}-\tau_{\varepsilon}\right| .
$$

We apply Lemma 3.2 with $2 S=2 R+n \delta$. Since $z_{n}, a_{n} \in \mathbb{V}_{\varepsilon}(2 S) \subset \mathbb{B}(2 S)$, we have

$$
\left|\frac{\phi_{n+1}(z)}{\phi_{n}(z)}-1\right| \leq \frac{C}{\left|\tau_{\varepsilon}\right|(R+n \delta / 2)^{1+\sigma}} \leq \frac{C^{\prime}}{(n+1)^{1+\sigma}},
$$

where $C^{\prime}=2^{1+\sigma} C / \delta^{1+\sigma}$ and we may assume $R>\delta / 2$. Now set $P:=$ $\prod_{n \geq 1}\left(1+C^{\prime} / n^{1+\sigma}\right)$. Since $\left|\phi_{n+1}(z) / \phi_{n}(z)\right| \leq 1+C^{\prime} /(n+1)^{1+\sigma}$, we have

$$
\left|\phi_{n}(z)\right|=\left|\frac{\phi_{n}(z)}{\phi_{n-1}(z)}\right| \cdots\left|\frac{\phi_{1}(z)}{\phi_{0}(z)}\right| \cdot\left|\phi_{0}(z)\right| \leq P|z-a| .
$$

Hence

$$
\left|\phi_{n+1}(z)-\phi_{n}(z)\right|=\left|\frac{\phi_{n+1}(z)}{\phi_{n}(z)}-1\right| \cdot\left|\phi_{n}(z)\right| \leq \frac{C^{\prime} P}{(n+1)^{1+\sigma}} \cdot|z-a| .
$$

This implies that $\phi_{\varepsilon}=\phi_{0}+\left(\phi_{1}-\phi_{0}\right)+\cdots=\lim \phi_{n}$ converges uniformly on compact subsets of $\mathbb{V}_{\varepsilon}(2 R)$ and for all $\varepsilon \in[0,1]$. The univalence of $\phi_{\varepsilon}$ is shown in the same way as [Mi, Lemma 10.10].

Next we claim that $\phi_{\varepsilon}\left(f_{\varepsilon}(z)\right)=\tau_{\varepsilon} \phi_{\varepsilon}(z)+B_{\varepsilon}$ with $B_{\varepsilon} \rightarrow 1$ as $\varepsilon \rightarrow 0$. One can easily check that $\phi_{n}\left(f_{\varepsilon}(z)\right)=\tau_{\varepsilon} \phi_{n+1}(z)+B_{n}$ where

$$
B_{n}=\frac{a_{n+1}-a_{n}}{\tau_{\varepsilon}^{n}}=\frac{\left(\tau_{\varepsilon}-1\right) a_{n}}{\tau_{\varepsilon}^{n}}+\frac{1+g_{\varepsilon}\left(a_{n}\right)}{\tau_{\varepsilon}^{n}} .
$$

When $\tau_{\varepsilon}=1, B_{n}$ tends to 1 since

$$
\left|g_{\varepsilon}\left(a_{n}\right)\right| \leq \frac{M}{\left|a_{n}\right|^{\sigma}} \leq \frac{M}{(2 R+n \delta)^{\sigma}} \leq \frac{M}{(n \delta)^{\sigma}} \rightarrow 0 .
$$


When $\left|\tau_{\varepsilon}\right|>1$, the last term of the formula for $B_{n}$ above tends to 0 . For $n \geq 1$, we have

$$
a_{n}=\tau_{\varepsilon}^{n} a+\frac{\tau_{\varepsilon}^{n}-1}{\tau_{\varepsilon}-1}+\sum_{k=0}^{n-1} \tau_{\varepsilon}^{n-1-k} g_{\varepsilon}\left(a_{k}\right)
$$

Thus

$$
\frac{\left(\tau_{\varepsilon}-1\right) a_{n}}{\tau_{\varepsilon}^{n}}=\left(\tau_{\varepsilon}-1\right)\left(a+\frac{g_{\varepsilon}(a)}{\tau_{\varepsilon}}+\sum_{k=1}^{n-1} \frac{g_{\varepsilon}\left(a_{k}\right)}{\tau_{\varepsilon}^{k+1}}\right)+1-\frac{1}{\tau_{\varepsilon}^{n}} .
$$

By the inequality on $\left|g_{\varepsilon}\left(a_{n}\right)\right|$ above, we have

$$
\left|\left(\tau_{\varepsilon}-1\right) \sum_{k=1}^{n-1} \frac{g_{\varepsilon}\left(a_{k}\right)}{\tau_{\varepsilon}^{k+1}}\right| \leq \frac{M}{\delta^{\sigma}} \frac{\left|\tau_{\varepsilon}-1\right|}{\left|\tau_{\varepsilon}\right|} \sum_{k=1}^{n-1} \frac{1}{k^{\sigma}\left|\tau_{\varepsilon}\right|^{k}} \leq \frac{M}{2 \delta^{1+\sigma}}\left(1-\frac{1}{\left|\tau_{\varepsilon}\right|}\right) \operatorname{Li}_{\sigma}\left(\frac{1}{\left|\tau_{\varepsilon}\right|}\right)
$$

where we have used the inequality

$$
\left|\tau_{\varepsilon}-1\right| \leq \frac{\operatorname{Re} \tau_{\varepsilon}-1}{\cos \alpha} \leq \frac{\left|\tau_{\varepsilon}\right|-1}{2 \delta}
$$

that comes from the radial convergence. By Proposition 4.1 in the next section, $B_{n}$ converges to some $B_{\varepsilon}$. More precisely, if we set $\left|\tau_{\varepsilon}\right|=e^{L}$, then $\tau_{\varepsilon}-1=O(L)$ and one can check that $B_{\varepsilon}=1+O\left(L^{\sigma /(1+\sigma)}\right)$.

Finally, $u_{\varepsilon}(z):=\phi_{\varepsilon}(z) / B_{\varepsilon}$ gives the desired holomorphic map (with $R$ in the statement replaced by $2 R$ ).

REMARKS.

- When $\sigma=1$, we have

$$
\left|\sum_{k=1}^{n-1} \frac{g_{\varepsilon}\left(a_{k}\right)}{\tau_{\varepsilon}^{k+1}}\right| \leq \frac{M}{\delta\left|\tau_{\varepsilon}\right|} \sum_{k=1}^{n-1} \frac{1}{k\left|\tau_{\varepsilon}\right|^{k}} \leq-\frac{M}{\delta} \log \left(1-\frac{1}{\left|\tau_{\varepsilon}\right|}\right)
$$

and this implies that $B_{\varepsilon}=1+O(L|\log L|)$ if we set $\left|\tau_{\varepsilon}\right|=e^{L}$. This is consistent with the result in [Ue1].

- By this proof, if $\left\{f_{\varepsilon}(z)\right\}$ analytically depends on $\varepsilon$, then so do $\left\{B_{\varepsilon}\right\}$ and $\left\{u_{\varepsilon}(z)\right\}$ for fixed $a$ in $\Pi(2 R)$.

- It is not difficult to check that $u_{\varepsilon}(z)=z\left(B_{\varepsilon}^{-1}+o(1)\right)$ as $z \rightarrow \infty$ within $\mathbb{V}_{\varepsilon}(R)$. (It is well-known that if $f_{0}(z)=z+1+a_{0} / z+\cdots$ then the Fatou coordinate is of the form $u_{0}(z)=z-a_{0} \log z+O(1)$. See [Sh].)

4. An estimate on polylogarithm functions. We define the polylogarithm function of exponent $s \in \mathbb{C}$ by

$$
\operatorname{Li}_{s}(z):=\sum_{n=1}^{\infty} \frac{z^{n}}{n^{s}} .
$$


This function makes sense when $|z|<1$ and $\sigma:=\operatorname{Re} s>0$ and it is a holomorphic function of $z$. In particular, if $\operatorname{Re} s>1$ the function tends to $\zeta(s)$ as $z \rightarrow 1$ within the unit disk. In the following we consider the behavior of $\operatorname{Li}_{s}(z)$ as $z \rightarrow 1$ within the unit disk when $0<\sigma \leq 1$. We claim:

Proposition 4.1. Suppose $0<\operatorname{Re} s=\sigma \leq 1$ and $z \rightarrow 1$ with $|z|<1$. Set $\varepsilon:=1-|z|$. Then there exists a uniform constant $C$ independent of $s$ such that

$$
\left|\operatorname{Li}_{s}(z)\right| \leq C \varepsilon^{-1 /(1+\sigma)}
$$

as $z \rightarrow 1$. In particular,

$$
\left|(z-1) \operatorname{Li}_{s}(z)\right| \leq C \varepsilon^{\sigma /(1+\sigma)} \rightarrow 0
$$

as $z \rightarrow 1-0$ along the real axis.

Proof. Clearly $\left|\operatorname{Li}_{s}(z)\right| \leq \sum_{n=1}^{\infty}|z|^{n} / n^{\sigma}$ so it is enough to consider the sum

$$
S:=\sum_{n=1}^{\infty} \frac{1}{n^{\sigma}} \cdot \lambda^{n}
$$

where $\lambda:=|z|=1-\varepsilon$. Let $S_{n}$ be the $n$th partial sum. By the Hölder inequality, we have

$$
S_{n} \leq\left(\sum_{k=1}^{n} \frac{1}{k^{\sigma p}}\right)^{1 / p}\left(\sum_{k=1}^{n} \lambda^{k q}\right)^{1 / q}
$$

for any $p, q>1$ with $1 / p+1 / q=1$. Now set $p:=1 / \sigma+1 \geq 2$ (then $1<q=1+\sigma \leq 2$ ). Since $\sigma p=1+\sigma>1$, the first sum is uniformly bounded:

$$
\sum_{k=1}^{n} \frac{1}{k^{\sigma p}} \leq 1+\int_{1}^{\infty} \frac{1}{x^{1+\sigma}} d x=1+\frac{1}{\sigma}=p .
$$

On the other hand, for the second sum, we still have $0<\lambda^{q}<1$ and thus

$$
\sum_{k=1}^{n} \lambda^{k q} \leq \frac{\lambda^{q}}{1-\lambda^{q}}=\frac{1}{q \varepsilon}(1+o(1)) \leq \frac{2}{q \varepsilon}
$$

when $\varepsilon \ll 1$. Hence we have the following uniform bound:

$$
S_{n} \leq p^{1 / p}\left(\frac{2}{q \varepsilon}\right)^{1 / q} \leq 2 \frac{p^{1 / p}}{q^{1 / q}} \varepsilon^{-1 / q} .
$$

One can easily check that $1 \leq x^{1 / x} \leq e^{1 / e}=1.44467 \ldots$ for $x \geq 1$. Thus

$$
S \leq 2 e^{1 / e} \varepsilon^{-1 / q}=2 e^{1 / e} \varepsilon^{-1 /(1+\sigma)}
$$

when $\varepsilon \ll 1$, and we have the desired estimate with $C=2 e^{1 / e}<3$. The last inequality of the statement follows from

$$
\left|(z-1) \operatorname{Li}_{s}(z)\right| \leq C \varepsilon^{1-1 / q}=C \varepsilon^{1 / p}=C \varepsilon^{\sigma /(1+\sigma)} .
$$

(Indeed, $\left|(z-1) \operatorname{Li}_{s}(z)\right|=O\left(\varepsilon^{\sigma /(1+\sigma)}\right)$ as $z \rightarrow 1$ radially.) 
5. Application: Proof of Theorem 1.2. As an application of Theorem 2.1, we give a proof of Theorem 1.2. Though Theorem 1.2 only deals with the simplest parabolic fixed point and its simplest perturbation, one can easily extend the result to general parabolic cycles with multiple petals and their "non-tangential" perturbations.

Proof of Theorem 1.2. Consider the general expression $f_{\lambda}(w)=\lambda w+w^{2}$ with $0<\lambda \leq 1$ (thus $f_{1}=g$ ). By looking at the action of $f_{\lambda}$ in a new coordinate $z=\chi_{\lambda}(w)=-\lambda^{2} / w$, we have

$$
\chi_{\lambda} \circ f_{\lambda} \circ \chi_{\lambda}^{-1}(z)=z / \lambda+1+O(1 / z)
$$

near $\infty$. Now we can set $\tau_{\varepsilon}:=1 / \lambda=1+\varepsilon$ and $f_{\varepsilon}:=\chi_{\lambda} \circ f_{\lambda} \circ \chi_{\lambda}^{-1}$ to have the same setting as in Theorem 2.1. We view $f$ and $g$ as being parameterized by $\lambda$ or $\varepsilon$. (It is convenient to use both parameterizations.) Note that $\Pi(R)=$ $\{\operatorname{Re} z \geq R\}$ in this case. By the same argument as in Lemma 3.1, we can check that $\operatorname{Re} f_{\varepsilon}(z) \geq \operatorname{Re} z+1 / 2$ if $z \in \Pi(R)$ and $R \gg 0$. In particular, $f_{\varepsilon}(\Pi(R)) \subset \Pi(R)$ for $R \gg 0$.

Let us show (1): For any compact $E \subset K_{g}^{\circ}$ and small $r>0$, there exists $N \gg 0$ such that $g^{N}(E) \subset P_{r}=\{|w+r| \leq r\}$. (For instance, one can deduce this from the existence of the Fatou coordinate.) By uniform convergence, we have $f^{N}(E) \subset P_{r}$ for all $f \approx g$. To prove $E \subset K_{f}^{\circ}$, it is enough to show that $f\left(P_{r}\right) \subset P_{r}$ for all $f \approx g$. Since $\chi_{\lambda}\left(P_{r}\right)=\Pi(R)$ for some $R \gg 0$, we have $f_{\varepsilon}(\Pi(R)) \subset \Pi(R)$ independently of $\varepsilon$. This is equivalent to $f_{\lambda}\left(P_{r}\right) \subset P_{r}$ in a different coordinate. Thus we have (1).

Next let us check (2): Set $\Phi_{\varepsilon}:=\Phi_{f}$ and $\Phi_{0}:=\Phi_{g}$. Then $\Phi_{\varepsilon}\left(f_{\lambda}(w)\right)=$ $\tau_{\varepsilon} \Phi_{\varepsilon}(w)+1$. On the other hand, by simultaneous linearization, we have uniform convergence $u_{\varepsilon} \rightarrow u_{0}$ on $\Pi(R)$ that satisfies $u_{\varepsilon}\left(f_{\varepsilon}(z)\right)=\tau_{\varepsilon} u_{\varepsilon}(z)+1$. By setting $\Psi_{\varepsilon}(w):=u_{\varepsilon} \circ \chi_{\lambda}(w)$, we have $\Psi_{\varepsilon} \rightarrow \Psi_{0}$ compact uniformly on $P_{r}$, and $\Psi_{\varepsilon}\left(f_{\lambda}(w)\right)=\tau_{\varepsilon} \Psi_{\varepsilon}(w)+1$.

We need to adjust the images of the critical orbits under $\Phi_{\varepsilon}$ and $\Psi_{\varepsilon}$. Since $g^{n}(-1 / 2) \rightarrow 0$ along the real axis, there is an $M \gg 0$ such that $g^{M}(-1 / 2)=$ : $a_{0} \in P_{r}$. By uniform convergence, we also have $f^{M}(-\lambda / 2)=: a_{\varepsilon} \in P_{r}$ and $a_{\varepsilon} \rightarrow a_{0}$ as $\varepsilon \rightarrow 0$. Set $b_{\varepsilon}:=\Psi_{\varepsilon}\left(a_{\varepsilon}\right)$ and $c_{\varepsilon}:=\Phi_{\varepsilon}\left(a_{\varepsilon}\right)$ for all $\varepsilon \geq 0$. Set also $\ell_{\varepsilon}(W)=\tau_{\varepsilon} W+1$. Then $c_{\varepsilon}=\ell_{\varepsilon}^{M}(0)=\tau_{\varepsilon}^{M-1}+\cdots+\tau_{\varepsilon}+1$ and $c_{\varepsilon} \rightarrow c_{0}=M$ as $\varepsilon \rightarrow 0$. When $\varepsilon>0$, we choose an affine map $T_{\varepsilon}$ that fixes $1 /\left(1-\tau_{\varepsilon}\right)$ and sends $b_{\varepsilon}$ to $c_{\varepsilon}$. When $\varepsilon=0$, we define $T_{0}$ to be the translation by $b_{0}-c_{0}$. Then one can check that $T_{\varepsilon} \rightarrow T_{0}$ compact uniformly on the plane and $\widetilde{\Phi}_{\varepsilon}:=T_{\varepsilon} \circ \Psi_{\varepsilon}$ satisfies $\widetilde{\Phi}_{\varepsilon} \rightarrow \widetilde{\Phi}_{0}$ on any compact subset of $P_{r}$. Moreover, $\widetilde{\Phi}_{\varepsilon}$ still satisfies $\widetilde{\Phi}_{\varepsilon}\left(f_{\lambda}(w)\right)=\tau_{\varepsilon} \widetilde{\Phi}_{\varepsilon}(w)+1$ and the images of the critical orbit under $\Phi_{\varepsilon}$ and $\widetilde{\Phi}_{\varepsilon}$ agree. Finally, by uniqueness of $\phi_{f}$ and $\phi_{g}$, one can check that $\Phi_{\varepsilon}=\widetilde{\Phi}_{\varepsilon}$ on $P_{r}$. 
Since

$$
\Phi_{f}(w)=\ell_{\varepsilon}^{-N} \circ \widetilde{\Phi}_{\varepsilon} \circ f^{N}(w) \rightarrow \ell_{0}^{-N} \circ \widetilde{\Phi}_{0} \circ g^{N}(w)=\Phi_{g}(w)
$$

uniformly on $E$, we have (2).

Acknowledgements. I would like to thank T. Ueda, F. Przytycki and the referee for correspondence. This research is partially supported by Inamori Foundation and JSPS.

\section{References}

[Ka] T. Kawahira, Tessellation and Lyubich-Minsky laminations associated with quadratic maps I: Pinching semiconjugacies, preprint, arXiv:math.DS/0609280.

[L] L. Leau, Étude sur les équations fonctionnelles à une ou à plusieurs variables, Ann. Fac. Sci. Toulouse 11 (1897), E.1-E.110.

[Mc] C. McMullen, Hausdorff dimension and conformal dynamics II: Geometrically finite rational maps Comment. Math. Helv. 75 (2000), 535-593.

[Mi] J. Milnor, Dynamics in One Complex Variable, 3rd ed., Ann. of Math. Stud. 160, Princeton Univ. Press, 2006.

[Sh] M. Shishikura, The Hausdorff dimension of the boundary of the Mandelbrot set and Julia sets, Ann. of Math. 147 (1998), 225-267.

[Ue1] T. Ueda, Schröder equation and Abel equation, preprint.

[Ue2] -, Simultaneous linearization of hyperbolic and parabolic fixed points, RIMS Kokyuroku 1494, 1-8.

Tomoki Kawahira

Graduate School of Mathematics

Nagoya University

Nagoya, 464-8602 Japan

E-mail: kawahira@math.nagoya-u.ac.jp 